\title{
An Extremely Simple Multi-Wing Chaotic System: Dynamics Analysis, Encryption Application and Hardware Implementation
}

\author{
Hairong Lin, Chunhua Wang, Fei Yu, Cong Xu, Qinghui Hong, Wei Yao and Yichuang Sun, Senior Member, \\ IEEE
}

\begin{abstract}
Polynomial functions have been the main barrier restricting the circuit realization and engineering application of multi-wing chaotic systems (MWCSs). To eliminate this bottleneck, we construct a simple MWCS without polynomial functions by introducing a sinusoidal function in a Sprott $\mathrm{C}$ system. Theoretical analysis and numerical simulations show that the MWCS can not only generate multi-butterfly attractors with an arbitrary number of butterflies, but also adjust the number of the butterflies by multiple ways including self-oscillating time, control parameters, and initial states. To further explore the advantage of the proposed MWCS, we realize its analog circuit using commercially available electronic elements. The results demonstrate that in comparison to traditional MWCSs, our circuit implementation greatly reduces the consumption of electronic components. This makes the MWCS more suitable for many chaos-based engineering applications. Furthermore, we propose an application of the MWCS to chaotic image encryption. Histogram, correlation, information entropy, and key sensitivity show that the simple image encryption scheme has high security and reliable encryption performance. Finally, we develop a field-programmable gate array (FPGA) test platform to implement the MWCS-based image cryptosystem. Both theoretical analysis and experimental results verify the feasibility and availability of the proposed MWCS.
\end{abstract}

Index Terms-Chaotic system, image encryption, FPGA implementation, nonlinear circuit, multi-butterfly attractor, multistability

\section{INTRODUCTION}

C HAOTIC behavior is a major discovery of human natural science in the 20th century, which has many specific properties like ergodicity, unpredictability, and initial state sensitivity [1]. Generally, dynamical systems with chaotic behaviors are called chaotic systems. Thanks to the chaotic properties, chaotic systems have been widely studied and applied in many real-world scenarios, such as weather prediction, industrial control, and market analysis [2-3]. In particular, they are often used in secure communication [45], as chaotic systems and cryptosystems have the same properties in unpredictability and initial state sensitivity. Since the well-known Lorenz chaotic system was discovered in 1963 [6], great efforts have been devoted to the development of

Manuscript received August 18, 2020; revised November 16, 2020. This work is supported by The Major Research Project of the National Natura Science Foundation of China (91964108), The National Natural Science Foundation of China $(61971185,61504013)$, The Open Fund Project of Key Laboratory in Hunan Universities (18K010). (Corresponding author: Chunhua Wang.)

Hairong Lin, Chunhua Wang, Cong Xu, Qinghui Hong and Wei Yao are with the College of Computer Science and Electronic Engineering, Hunan University, Changsha, 410082, China. (wch1227164@hnu.edu.cn)

Fei Yu is with School of Computer and Communication Engineering in Changsha University of Science and Technology, Changsha, 410082, China

Yichuang Sun is with the School of Engineering and Computer Science, University of Hertfordshire, Hatfield AL10 9AB, U.K. various new chaotic systems [7-8], such as multi-scroll chaotic systems (MSCSs) [9], multi-wing chaotic systems (MWCSs) [10], hyperchaotic systems [11], and chaotic systems with multistability [12]. Among them, the MSCSs and MWCSs have more flexible adjustability and higher complexity, which makes them have a more promising application for information encryption [13-14]. As we all know, a common method to construct MSCSs is based on the double-scroll Chua's circuit model [9]. That is to say, the design of MSCSs is not a difficult task. But MWCSs are hard to be realization based on the double-wing Lorenz-like system in the same way done in Chua's circuit. This is because the nonlinearity of the Lorenz-like system comes from its cross-product terms or square terms. Thus, the design of MWCSs is still challenging work.

Over the past few decades, there have been many methods to develop MWCSs owning multi-wing attractors. In 2002, Elwakil et al. [15] constructed the first four-wing chaotic system by introducing one binary switch in a modified Lorenz system. One year later, Chen and Liu [16] obtained another four-wing butterfly attractor from a new Lorenz-like system. From then on, the realization of MWCSs has greatly stimulated the researcher's interest, and a large number of different types of MWCSs have been proposed [17-18]. During this period, Yu et al. presented many important theories on the construction of MWCSs and successively put forward a series of nonlinear polynomial function control methods including a multisegment quadratic function method [19], symmetric piecewise-linear function method [20], and switching control function method [21]. The basic idea is that the nonlinear polynomial function would increase the number of index-2 equilibrium points where the wings could be produced. In recent years, more and more MWCSs have been presented by using other polynomial functions, for example, state feedback controller function [22], piecewise hysteresis function [23], sign function [24], and so on [25-26]. Nevertheless, in these efforts, there are three major drawbacks: (i) Both the system model and algorithm are very complex, as the nonlinear function has cumulative polynomials; (ii) It is inconvenient to adjust the number of the wings because of the mutual effect of multiple control parameters; (iii) The implementation of the circuit is more and more complicated with the increase of wings.

Recently, numerous efforts are devoted to developing new MWCSs via using non-function control methods [27]. Particularly, by applying a multi-level pulse control method, Hong et al. [28-29] proposed multiple new MWCSs with multibutterfly attractors. Such method significantly simplifies the circuit realization of multi-wing-type chaotic systems because 
of not using nonlinear polynomial functions. However, the drawback in the system model and algorithm has not been fully overcome, as the used multi-level pulse has also multiple control parameters. Furthermore, to obtain more wings, multiple pulse signal generators with different levels are required simultaneously, which greatly increases the difficulty of hardware circuit implementation.

In fact, the physical implementation of chaotic systems is important and necessary to the chaos application. Up to now, some MWCSs mentioned above have been physically realized by using analog circuits or field-programmable gate array (FPGA). However, these implementations are quite difficult because of the existence of polynomial functions with multiple control parameters. Particularly, both their circuits and algorithms will become more and more complex with the increase of wings or butterflies. Therefore, finding a novel MWCS that can avoid the existence of polynomial functions is still a challenging and significant research topic.

This paper proposes an extremely simple MWCS without any polynomial functions. Since the new system has no polynomial functions, its mathematical model, algorithm, and circuit are greatly simplified. Theoretical analysis, practical application, and experimental results demonstrate the excellent performances of the proposed MWCS. This paper main includes two theoretical novelties and one experimental contribution:

1) An extremely simple MWCS with no polynomial functions is designed, and its unique dynamics properties are revealed.

2) A MWCS-based image encryption scheme is proposed, and its various security metrics are analyzed.

3) The image cryptosystem based on the MWCS is implemented and demonstrated on the FPGA platform.

The rest of this paper is arranged as follows. Section II presents a MWCS and investigates its dynamical properties. Section III realizes the analog circuit of the MWCS. Section IV proposes a MWCS-based image encryption scheme. Section V implements the MWCS-based image cryptosystem on the FPGA platform.

\section{SYSTEM INTRODUCTION AND DYNAMICS ANALYSIS}

In general, the realization of multi-wing attractors must be based on a double-wing Lorenz-like chaotic system. Here we construct a new MWCS based on the famous Sprott C system with a double-wing butterfly attractor [30]. The MWCS is described as

$$
\left\{\begin{array}{l}
\dot{x}=a(y-x) \\
\dot{y}=b x \sin (z) \\
\dot{z}=c-y^{2}
\end{array}\right.
$$

where $x, y, z$ are three state variables, and $a, b, c$ are three positive system parameters. It is evident that system (1) has only five terms containing two nonlinear terms $b x \sin (z)$ and $y^{2}$, and has no extended polynomial functions. Therefore, the proposed system enjoys an extremely simple mathematical model as compared with the existing MWCSs that own polynomial functions. In the following subsections, we will try to study its dynamics properties for exhibiting its uniqueness.

\section{A. Stability for Infinite Discrete Equilibrium Points}

In this subsection, according to the heteroclinic loop theorem [28], the equilibrium points and their stabilities are revealed by theoretical analysis and graph description. In system (1), letting $\dot{x}=\dot{y}=\dot{z}=0$, the equilibrium points of the system can be computed as

$$
E=\left(x^{*}= \pm \sqrt{c}, y^{*}= \pm \sqrt{c}, z^{*}=k \pi\right)
$$

where $k=0, \pm 1, \pm 2, \ldots$ Clearly, the presented MWCS has infinite discrete equilibrium points. And the stability of all the equilibrium points can be determined by eigenvalues of following the Jacobin matrix.

$$
J=\left[\begin{array}{lll}
\frac{\partial \dot{x}}{\partial x} & \frac{\partial \dot{x}}{\partial y} & \frac{\partial \dot{x}}{\partial z} \\
\frac{\partial \dot{y}}{\partial x} & \frac{\partial \dot{y}}{\partial y} & \frac{\partial \dot{y}}{\partial z} \\
\frac{\partial \dot{z}}{\partial x} & \frac{\partial \dot{z}}{\partial y} & \frac{\partial \dot{z}}{\partial z}
\end{array}\right]=\left[\begin{array}{lll}
-a & a & 0 \\
b \sin (z) & 0 & b x \cos (z) \\
0 & -2 y & 0
\end{array}\right]
$$

Substituting Equation (2) into Equation (3), we get

$$
J_{1 / 2}=\left[\begin{array}{lll}
-a & a & 0 \\
0 & 0 & \pm b \sqrt{c} \cos (k \pi) \\
0 & \mp 2 \sqrt{c} & 0
\end{array}\right] .
$$

Both $J_{1}$ and $J_{2}$ have the same characteristic equation:

$$
(\lambda+a)\left(\lambda^{2}+2 b c \cos (k \pi)\right)=0 .
$$

In Equation (5), there are different eigenvalues under different parity of $k$. If $k$ is an odd number, the eigenvalues of Equation (5) can be given by

$$
\lambda_{1}=-a, \lambda_{2}=\sqrt{2 b c}, \lambda_{3}=-\sqrt{2 b c} .
$$

Consequently, in this case, the corresponding equilibrium point in Equation (2) is an unstable index-1 saddle point. If $k$ is an even number, the eigenvalues of Equation (5) can be obtained by

$$
\lambda_{1}=-a, \lambda_{2}=\sqrt{2 b c} i, \lambda_{3}=-\sqrt{2 b c} i .
$$

So, in this case, the corresponding equilibrium point in Equation (2) is a nonhyperbolic equilibrium point.

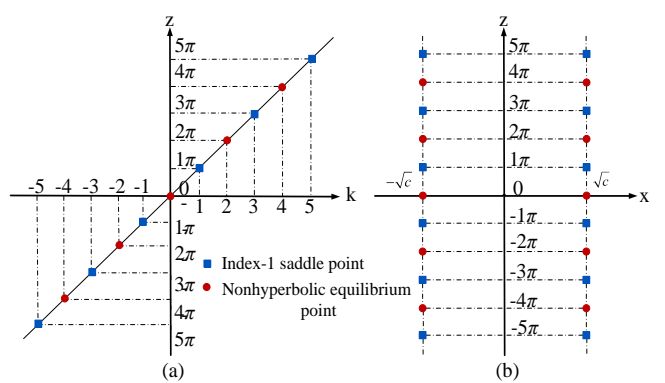

Fig. 1: Equilibrium points distribution of system (1). (a) $k-z$ plane. (b) $x-z$ plane.

Next, the distribution of the equilibrium points is discussed by graph description. In Equation (2), the equilibrium points of the system are determined by the equation $z=k \pi$. Namely, the equilibrium points in the $z$-axis will orderly extend with the increase of $k$. It should be pointed that unlike the previous MWCSs, all equilibrium points in system (1) exist in the system itself, rather than being extended by adding additional polynomial functions. Thus, the new system does not require additional polynomial functions to yield multibutterfly attractors. Let $k=0, \pm 1, \pm 2, \pm 3, \pm 4, \pm 5$, Fig. 1 illustrates the distribution of the equilibrium points on the $k$ $z$ plane and $x-z$ plane with the increase of $k$. We can see that the equilibrium points of system (1) are extended along the $z$-axis with the increase of $k$. And all equilibrium points are symmetric about the $z$-axis, which forms infinite pairs of equilibrium points with the same stabilities. Moreover, the 
nonhyperbolic equilibrium points and the unstable index-1 saddle points are alternately extended along $z$-axis direction in the $x-z$ plane.

\section{B. Parameter c-dependent Bifurcation Dynamics}

The above subsection shows that system (1) has infinitely many equilibrium points that are related to the parameter $c$. In this subsection, the dynamic characteristics of $c$ are investigated by using bifurcation diagram and Lyapunov exponents. Set the parameters $a=1, b=2.1$, the initial values $\left(x_{0}\right.$, $\left.y_{0}, z_{0}\right)=(0.1,0.1,0.1)$, we can plot the bifurcation diagrams and Lyapunov exponents of system (1) with respect to the parameter $c \in(0,1.8)$, as shown in Fig. 2, where $x \max$ is the maxima of the $x$ variable. Fig. 2(a) shows that system (1) appears forward period-doubling bifurcation with the increase of parameter $c$. When $c$ increases to 0.055 , system (1) enter into an intermittent chaotic area until $c=0.62$. With the $c$ further increase, the chaotic state is degenerated to a periodic state by tangent bifurcation. However, the periodic state quickly evolves into a stable chaotic area by forward period-doubling bifurcation route again. Finally, the chaotic state degenerates to a stable point through the tangent bifurcation route at $c=1.7$. The Lyapunov exponents in Fig. 2(b) are basically consistent with the dynamical behavior on the bifurcation in Fig. 2(a). The phase portraits of the attractors of the system (1) with different values of $c$ are further presented to illustrate its dynamic evolution with the parameter, as shown in Fig. 3. It is obvious that system (1) generates different attractors from initial values $(0.1,0.1,0.1)$, for $c=0.048,0.051,0.01,1.2$, respectively.

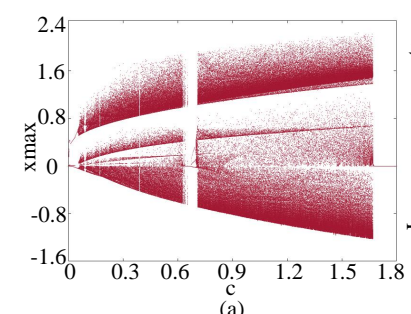

(a)

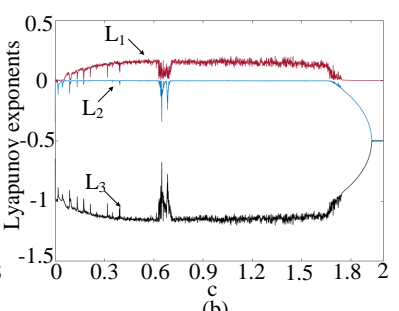

(b)
Fig. 2: Bifurcation diagrams and Lyapunov exponents of system (1) for $a=1, b=2.1, c \in(0,1.8)$. (a) Bifurcation diagram. (b) Lyapunov exponents.

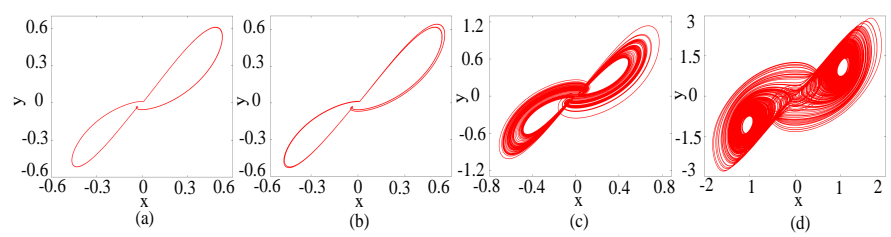

Fig. 3: Phase portraits of attractors in system (1) with $a=1$, $b=2.1$, and different parameter $c$. (a) Period-1 attractor with $c=0.048$. (b) Period- 2 attractor with $c=0.051$. (c) Chaotic attractor with $c=0.1$. (d) Chaotic attractor with $c=1.2$.

\section{Self-oscillating Time-dependent Multi-butterfly Attractors}

Assuming that the system parameters $a=1, b=2.1$ and $c=0.45$, initial states $\left(x_{0}, y_{0}, z_{0}\right)=(0.3,0.3,-14)$, and simulation time $t s=800$ time units, we can obtain a basic doublewing butterfly attractor by solving system (1) with the ODE45 algorithm in Matlab R2017a, as shown in Fig. 4. Interestingly, the trajectory of the basic butterfly attractors is attracted by a pair of nonhyperbolic equilibrium points, which is different from some traditional butterfly attractors around unstable index-2 saddle focus.
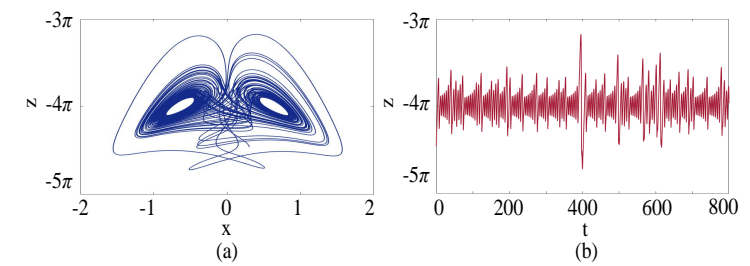

Fig. 4: Basic double-wing butterfly attractor of system (1) with system parameters $a=1, b=2.1, c=0.45$, initial states $\left(x_{0}\right.$, $\left.y_{0}, z_{0}\right)=(0.3,0.3,-14)$, and simulation time $t s=800$. (a) Phase portrait on the $x-z$ plane; (b) Time series of state variable $z$.

It is more interesting that extensive simulation results show that the basic butterfly attractor is orderly duplicated with the increase of the time of the system self-oscillating. That is to say, system (1) can generate multi-butterfly attractors, where the number of butterflies is dependent on the simulation time of the system. Keeping the system parameters and initial states unchanged, the different number of multi-butterfly attractors can be observed under different simulation time, as shown in Fig. 5. Fig. 5 directly illustrates two important phenomena: (i) system (1) generates multi-butterfly attractors and (ii) The longer simulation time comes along with more butterflies in a multi-butterfly attractor. As we can see, the phase space of the system is extended to multiple parts along the $z$-axis with the increase of simulation time, in which each part represents a basic butterfly attractor. That is, each pair of nonhyperbolic equilibrium points form a heteroclinic orbit and correspond to an independent butterfly attractor. Furthermore, unstable saddle points are located outside of the butterfly attractors, which plays a key role in connecting two independent butterfly attractors. The reason for changing simulation time to generate a different number of multi-butterfly attractors is that the nonlinearity of the system is unbound. The time series of state variable $z$ given in Fig. 6(a) further illustrates this result. In Fig. 6(a), the value of $z$ increases as the simulation time $t s$ increases, which leads to a bigger number of multi-butterfly attractors. Furthermore, the corresponding first three Lyapunov exponents are given in Fig. 6(b) to further demonstrate the chaotic properties of the system. Evidently, the largest Lyapunov exponent in Fig. 6(b) is always positive, which shows that system (1) generates chaotic behavior.
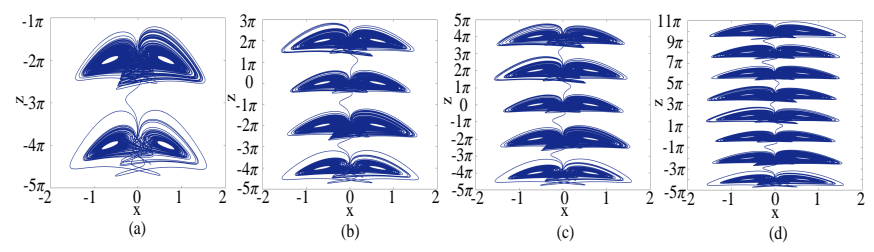

Fig. 5: Multi-butterfly attractors obtained by setting different simulation time $t s$. (a) 2-butterfly attractor with $t s=2500$. (b) 4-butterfly attractor with $t s=5500$. (c) 5-butterfly attractor with $t s=6500$. (d) 8-butterfly attractor with $t s=10000$.

\section{Control Parameters-dependent Multi-butterfly Attractors}

Although the multi-butterfly attractors with an arbitrary number of butterflies could be obtained in the system (1) by selecting appropriate transient calculation time, it is not convenient in practical applications to choose a different 

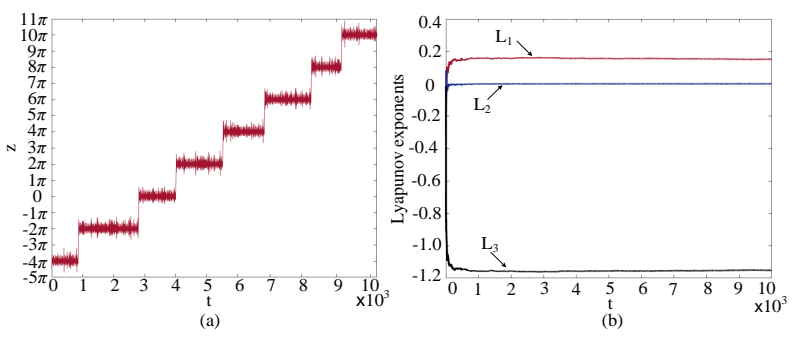

Fig. 6: Chaotic properties of system (1) with $t s=10000$. (a)Time series of state variable $z$. (b) Lyapunov exponents of system (1).

number of multi-butterfly attractor by controlling the system self-oscillating time. Consequently, we design an additional controller to accurately control the number of multi-butterfly attractors. Noted that the number of multi-butterfly attractors changes with transient simulation time, which is caused by the unboundedness of state variable $z$ in the system. So, the number of butterflies can be controlled by adding a bounded function to bound the range of state variable $z$ in the system. Based on this strategy, a simple bounded function can be designed by $H(z)=\operatorname{sgn}(z+d)+\operatorname{sgn}(z-e)$, where $d$ and $e$ are two positive control parameters, and sgn is the signum function. As a result, the improved system can be described by

$$
\left\{\begin{array}{l}
\dot{x}=a(y-x) \\
\dot{y}=b x \sin (z)+(\operatorname{sgn}(z+d)+\operatorname{sgn}(z-e)) \\
\dot{z}=c-y^{2}
\end{array} .\right.
$$

Fortunately, the number of multi-butterfly attractors can be determined by the control parameter $d$ and $e$. Keeping system parameters $a=1, b=2.1, c=0.45$, initial states $(0.3,0.3,-14)$, and transient simulation time $t s=10000$ unchanged, multibutterfly attractors with different number of butterflies can be obtained from system (8) via setting appropriate control parameter values, as shown in Fig. 7. It can be seen clearly from Fig. 7(b) and (d) that the value of the state variable $z$ increases with the simulation time, but it is restricted within the range $d<z<e$ and the value does not jump and exceed $e$. As a result, the phase space of the multi-butterfly attractors is limited within the range $d<z<e$ in the $z$ axis direction. Also, the number of the multi-butterfly attractors will meet $n=$ floor $((d+e) / 2 \pi)$, where floor rounds the elements of $x$ to the nearest integers less than or equal to $x$.

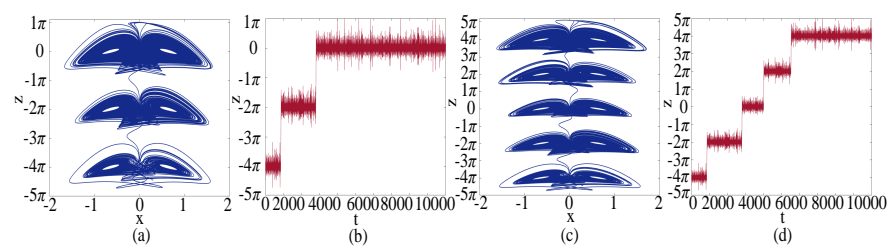

Fig. 7: Multi-butterfly attractors obtained by selecting different values of control parameters $d, e$, and the corresponding time series of state variable $z$. (a) 3-butterfly attractor with $d=18$ and $e=3$. (b) time series of the 3-butterfly attractor. (c) 5-butterfly attractor with $d=18$ and $e=16$. (d) time series of the 5-butterfly attractor.

\section{E. Initial States-dependent Multi-butterfly Attractors}

Multistability is a kind of special dynamical phenomenon that represents the coexistence of multiple different attractors in dynamical systems under different initial states [31].
Although multistability is found in many chaotic systems [32-33], the MWCSs with multistability have not been reported. In this subsection, the multistability of the proposed MWCS is discorvered. When keeping system parameters $a=1$, $b=2.1, c=0.45$, control parameters $d=18, e=16$, simulation time $t s=10000$ unchanged, coexisting multiple multi-butterfly attractors can be obtained from the following five different initial states $(0.3,0.3,-14),(0.3,0.3,-6),(0.3,0.3,0)$, $(0.3,0.3,6)$, and $(0.3,0.3,14)$, as shown in Fig. 8. Fig. 8 shows that the constructed MWCS exhibits coexisting multibutterfly attractors, namely, multistability. Also, the number of butterflies is reduced with the increase of initial value $z_{0}$. That is, the number of multi-butterfly attractors can be adjusted by changing initial states. And we can confirm that more coexisting multi-butterfly attractors with different number of butterflies will be found by selecting larger control parameter range $(d, e)$ and more initial states. Furthermore, when control parameters are selected as $d=18, e=35$, and both $x_{0}$ and $y_{0}$ are kept as 0.3 , the bifurcation diagram of system (8) concerning $z_{0} \in(-15,35)$ are plotted for further illustrating the coexistence of multi-butterfly attractors in Fig. 9. Fig. 9 directly shows that the system generates multiple multibutterfly attractors with different number of butterflies with the increase of $z_{0}$, which means that the proposed MWCS has complex multistability.

Table I provides a summary of the comparison between different MWCSs reported in recent years. It can be seen from the second and the third column of Table I that all existing MWCSs have at least one polynomial function/pulse. On the contrary, the proposed MWCS has no polynomial functions, which means that it enjoys a very simple system model. In the fourth column of Table I, there are at least $i$ control parameters in the existing MWCS, where $i$ represents the number of wings or butterflies. On the contrary, no matter how many the number of butterflies is generated, the presented MWCS has only two control parameters. That is to say, the number of control parameters in this MWCS does not increase with the increase in the number of butterflies. Moreover, the fifth column of Table I illustrates that the proposed MWCS is the first MWCS with multistability.

\section{CiRCUit VERIFICATION AND PERFORMANCE COMPARISION}

The electronic circuit design is considered as an important method to analyze the chaotic systems. It can physically verify the existence of chaotic attractors and provide the possibility for their engineering applications. The algebraic forms of the conventional MWCSs with polynomial functions are quite complex. Hence, it is very difficult to physically realize their multi-wing chaotic attractors by using the electronic circuit. On the contrary, since the proposed MWCS enjoys a simple algebraic expression, an electronic circuit can be easily designed to implement its multi-wing chaotic attractors. In this section, the MWCS is physically realized and experimentally analyzed.

\section{A. Multi-Wing Chaotic Circuit implementation}

It is of great significance to verify chaos with analog circuits because analog chaotic electronic circuits can generate chaos in the true sense. In general, the nonlinear dynamical equations can be physically realized by using basic passive or active electronic elements [34]. Here, the analog circuit of 
TABLE I: COMPARISON OF DIFFERENT MULTI-WING CHAOTIC SYSTEMS

\begin{tabular}{|c|c|c|c|c|}
\hline Refs & System model & Polynomial function/pulse & $\begin{array}{c}\text { Control } \\
\text { parameters }\end{array}$ & Multistability \\
\hline 2015 [22] & $\left\{\begin{array}{l}\dot{x}=a(y-x) \\
\dot{y}=-z \operatorname{sgn}(x)+b w \\
\dot{z}=f(x)-1 \\
\dot{w}=-c y\end{array}\right.$ & $f(x)=k|x|-\sum_{i=1}^{N} F_{i}\left(2+\operatorname{sgn}\left(x-E_{i}\right)-\operatorname{sgn}\left(x+E_{i}\right)\right)$ & $F_{i}, E_{i}$ & No \\
\hline $2016[25]$ & $\left\{\begin{array}{l}\dot{x}=\alpha y-\rho W(\varphi) x \\
\dot{y}=\beta y-x z / P \\
\dot{z}=f(x)-\gamma z \\
\dot{\varphi}=x\end{array}\right.$ & $f(x)=F_{0} x^{2}-\sum_{i=1}^{N} F_{i}\left(10.5 \operatorname{sgn}\left(x-E_{i}\right)-0.5 \operatorname{sgn}\left(x+E_{i}\right)\right)$ & $F_{i}, E_{i}$ & No \\
\hline $2018[24]$ & $\left\{\begin{array}{l}\dot{x}=a(y-x) \\
\dot{y}=x z-x u \\
\dot{z}=b-y^{2} \\
\dot{u}=f(z)-u\end{array}\right.$ & $f(z)=\sum_{i=1}^{n} l_{i} \operatorname{sgn}\left(z+p_{i}\right)$ & $l_{i}, p_{i}$ & No \\
\hline 2019 [28] & $\left\{\begin{array}{l}\dot{x}=a(y-f(x)) \\
\dot{y}=\operatorname{sgn}(f(x))(b-z) \\
\dot{z}=|f(x)|\end{array}\right.$ & $f(x)=x-\sum_{i=1}^{N} A_{i} \operatorname{sgn}\left(\sin \left(w_{i} t-\phi_{i}\right)\right)$ & $A_{i}, w_{i}, \phi_{i}$ & No \\
\hline This work & $\left\{\begin{array}{l}\dot{x}=a(y-x) \\
\dot{y}=b x \sin (z) \\
\dot{z}=c-y^{2}\end{array}\right.$ & No & $d, e$ & Yes \\
\hline
\end{tabular}

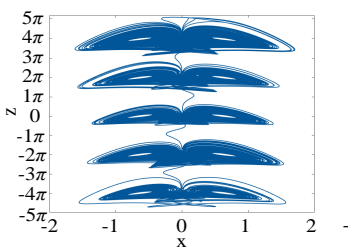

(a)

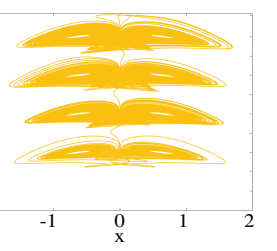

(b)

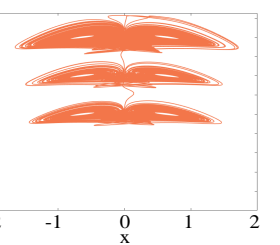

(c)

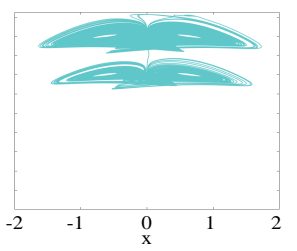

(d)

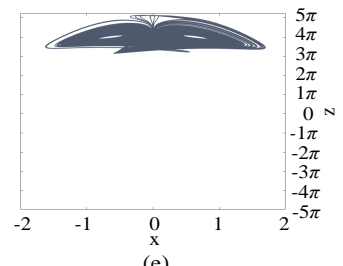

(e)

Fig. 8: Five coexisting multi-butterfly attractors under different initial states. (a) 5-butterfly attractor with initial state $(0.3$, $0.3,-14)$. (b) 4-butterfly attractor with initial state $(0.3,0.3,-6)$. (c) 3-butterfly attractor with initial state $(0.3,0.3,0)$. (d) 2-butterfly attractor with initial state $(0.3,0.3,6)$. (e) 1-butterfly attractor with initial state $(0.3,0.3,14)$.

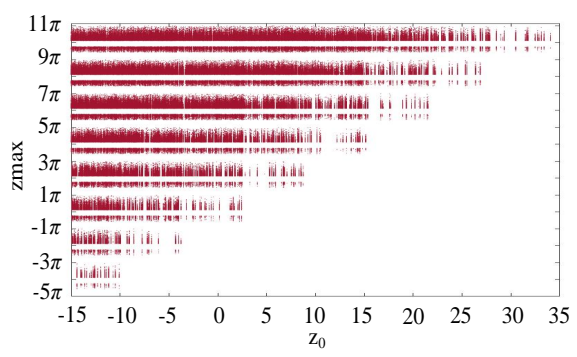

Fig. 9: Bifurcation diagram of the MWCS with $a=1, b=2.1$, $c=0.45, d=18, e=35, x_{0}=y_{0}=0.3$, and $z_{0} \in(-15,35)$.

the proposed MWCS is implemented by adopting capacitors, resistors, operational amplifiers TL082CP, analog multipliers AD633JN and trigonometric function converters (TFC) AD639AD all powered by $\pm 18 \mathrm{~V}$ symmetric voltages.

Before realizing the system circuit, we first design a control circuit, as shown in Fig. 10(a). In Fig. 10(a), when $R_{S}=13.5 \mathrm{k} \Omega, R_{F}=1 \mathrm{k} \Omega$, and $R_{L}=10 \mathrm{k} \Omega$, the input-output relationship of the control circuit can be expressed by

$$
H\left(v_{i n}\right)=-\frac{13.5 R_{F}}{R_{S} R_{L}}\left(\operatorname{sgn}\left(v_{i n}+v_{d}\right)+\operatorname{sgn}\left(v_{i n}-v_{e}\right)\right),
$$

where $v_{i n}$ is the input voltage, $v_{d}$ and $v_{e}$ simulate two control parameters which can be changed according to practical need. Considering the system (1), the multi-wing chaotic circuit is designed in Fig. 10(b). It is noted that a switch is added in the multi-wing circuit to control the control circuit. According to
Kirchhoff circuit law, the corresponding circuit state equations are given by

$$
\left\{\begin{array}{l}
R C \frac{d v_{x}}{d t}=\frac{R}{R_{2}} v_{y}-\frac{R}{R_{1}} v_{x} \\
R C \frac{d v_{y}}{d t}=\frac{R}{R_{5}} g v_{x} \sin \left(v_{z}\right)+R H\left(v_{z}\right), \\
R C \frac{d v_{z}}{d t}=\frac{R}{R_{6}} v_{c}-\frac{R}{R_{7}} g\left(v_{y}\right)^{2}
\end{array}\right.
$$

where $R C$ is the integral time constant, $g=1$ is the control gain of the multiplier $\mathrm{M}$, and $v_{x}, v_{y}$, and $v_{z}$ are the capacitor voltages of three integral circuits, respectively. Assuming that $R C=1 \mathrm{~ms}$, the resistance $R=10 \mathrm{k} \Omega$, then the capacitance $C$ can be chosen as $100 \mathrm{nF}$. In view of the parameters of the system and error influence, resistances can be calculated as $R_{1}=R_{2}=R / a, R_{3}=R_{4}=10 \mathrm{k} \Omega, R_{5}=R / b, R_{6}=R_{7}=10 \mathrm{k} \Omega$. Furthermore, parameters $c, d$, and $e$ are simulated by using $v_{c}, v_{d}$, and $v_{e}$, respectively. The multi-wing chaotic circuit is implemented on the experimental circuit breadboard. Considering system parameters $a=1, b=2.1$, the resistors $R_{1}, R_{2}$, and $R_{5}$ are taken as $10 \mathrm{k} \Omega, 10 \mathrm{k} \Omega$, and $4.76 \mathrm{k} \Omega$, respectively. When the switch $\mathrm{K}$ is closed, $v_{c}$ is set as $0.45 \mathrm{~V}$, and the circuit simulation time is fixed as 10s, multi-butterfly attractors with different number of butterflies can be observed under initial capacitor voltages $(0.3 \mathrm{~V}, 0.3 \mathrm{~V},-14 \mathrm{~V})$ and different circuit control parameters $v_{d}$ and $v_{e}$. For example, when two sets of control parameters $\left(v_{d}, v_{e}\right)$ are selected as $(18 \mathrm{~V}, 3 \mathrm{~V})$ and $(18 \mathrm{~V}$, $16 \mathrm{~V}), 3$-butterfly attractor, and 5-butterfly attractor can be captured as shown in Fig. 11. It is clear that the chaotic circuit generates multi-butterfly attractors, which further verifies the above theoretical analysis and numerical simulation results. 


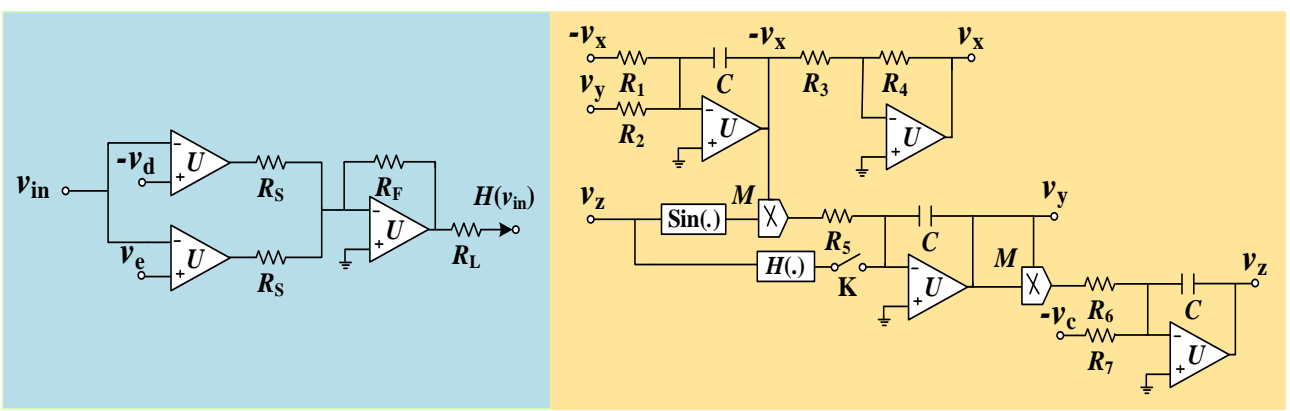

(a)

(b)

Fig. 10: Analog circuit implementation. (a) Circuit configuration of the control system. (b) Circuit configuration of the multiwing chaotic system.

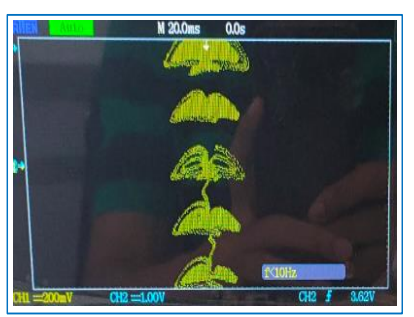

(a)

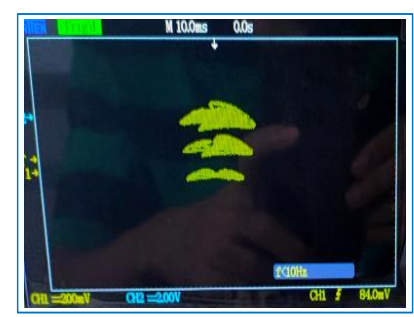

(b)
Fig. 11: Experimental results. (a) 3-butterfly attractor with $v_{d}=18 \mathrm{~V}$ and $v_{e}=3 \mathrm{~V}$. (b) 5-butterfly attractor with $v_{d}=18 \mathrm{~V}$ and $v_{e}=16 \mathrm{~V}$.

\section{B. Comparison of Related Multi-Wing Circuits}

Through the above circuit implementation, it is concluded that generating multi-wing attractor does not need to change the topology of the original double-wing circuit. The conversion of the number of butterflies can be realized by adjusting two input voltages $v_{d}$ and $v_{e}$. In the past several years, various circuits of polynomial functions are implemented by utilizing a large number of operational amplifiers. Hence, the implementation of multi-wing circuits is very complex. On the contrary, in our circuit, there is no need to design a complicated polynomial function circuit, which reduces difficulties in circuit realization. Also, in our circuit, the increase of each butterfly attractors correspond to the addition of the amplitude of the voltage. Therefore, the hardware implementation cost benefit of the presented architecture is obvious, which will reduce the hardware cost of the chaotic circuit in practical application. Table II presents the comparison between the circuit implementation of related MWCSs with $n$ wings or butterflies. In the table, CR stands circuit realization. It can be found from the column 3, 4, 6 of Table II that for [22, 24-26], the greater number $(n)$ of wings or butterflies is generated, the more operational amplifiers (Opm) and resistors $(\mathrm{R})$ are required. Although the number of operational amplifiers and resistors in [28] is unchanged with the increase of butterflies, the number of pulse generators (PG) is accordingly increased. In contrast, the designed multi-wing chaotic circuit requires fewer amplifiers and resistors, as compared with [22, 24-26, 28], reducing area and power dissipation. It also does not require pulse generators. Therefore, the circuit implementation of the new MWCS is significantly simplified. It means that the presented MWCS can be applied in more wide fields.
TABLE II: COMPARISON OF RELATED MULTI-WING CHAOTIC CIRCUITS

\begin{tabular}{|c|c|c|c|c|c|c|c|}
\hline Refs & CR & Attractor & Opm & M & R & PG & TFC \\
\hline$[22]$ & Yes & $n$-wing & $18+5 n$ & 1 & $33+5 n$ & 0 & 0 \\
\hline$[25]$ & Yes & $n$-wing & $7+5 n$ & 4 & $10+10 n$ & 0 & 0 \\
\hline$[24]$ & Yes & $n$-butterfly & $5+2 n$ & 3 & $9+2 n$ & 0 & 0 \\
\hline$[26]$ & No & $n$-butterfly & $13+n$ & 4 & $32+n$ & 0 & 0 \\
\hline$[28]$ & Yes & $n$-butterfly & 15 & 2 & 32 & $n$ & 0 \\
\hline This work & Yes & $n$-butterfly & 7 & 2 & 11 & 0 & 1 \\
\hline
\end{tabular}

\section{ApPliCATION IN IMAGE ENCRYPTION}

The study of chaos-based image encryption has been a recent focus [35-37]. Generally, systems with multiple wings or multistability have become potential candidates for chaos applications compared to chaotic systems with fewer wings or stability due to their complex dynamical properties. Thus, the proposed MWCS would have a more attractive application prospect in image encryption schemes, as it has both multi-butterfly attractors and multistability. In this section, an MWCS-based image encryption scheme is proposed to verify its merit for image secure communication.

\section{A. Description of Encryption Process}

As shown in Fig. 12, the architecture of the MWCS-based image encryption scheme mainly consists of four blocks: chaotic sequence generator, key generator, encryption module, and decryption module. We assumes that a grayscale original image $\mathrm{P}$ is used as the encryption object. The process of encryption and decryption is composed of four steps of operations.

1) Chaotic sequence generator: Set system parameters, control parameters, initial values, discarded number $N_{0}$, and time step, then iterate the MWCS with the fourthorder Runge-Kutta algorithm (ODE45). Three discrete chaotic sequences $(x(i), y(i), z(i))$ kept the same length with the original image are obtained.

2) Key generator: The three chaotic sequences are reprocessed as following:

$$
K(i)=\bmod \left(\text { floor }\left(((x(i)+y(i)+z(i)) / 3) * 10^{15}\right), 256\right),
$$

where the floor $(x)$ rounds the elements of $x$ to the nearest integers less than or equal to $x$.

3) Encryption module: The original image is encrypted as

$$
\boldsymbol{P}^{\prime}(i)=\boldsymbol{P}(i) \oplus K(i),
$$

where the symbol $\oplus$ represents the exclusive XOR operation.

4) Decryption module: The encrypted image is decrypted with the reverse process of the encryption algorithm. 


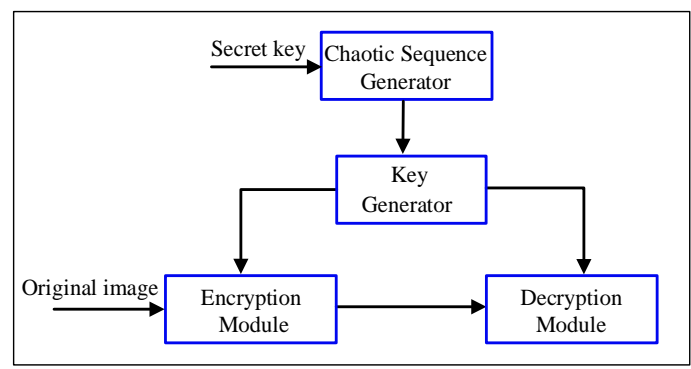

Fig. 12: The architecture of the MWCS-based image encryption scheme.

\section{B. Experiments and Performance Analyses}

To demonstrate the efficiency of the presented image encryption scheme, numerical simulation experiments were performed by Matlab R2017a. And some classic metrics like histogram, correlation coefficient, information entropy, and key sensitivity were measured [5, 13, 35-37]. In all experiments, the "Lena $256 \times 256$ " image is employed as the test image, as shown in Fig. 13(a). Setting $a=1, b=2.1, c=0.45$, $d=18, e=16$ and initial states $(0.3,0.3,-14)$, a pseudorandom sequence generated by Equation (11) is used for image encryption. In this process, to avoid the transient effect, the first $N_{0}=500$ results of the iteration are discarded, and the time step is chosen as 0.001 .

1) Histogram analysis: The histogram of an image represents the distribution of the pixel intensity values in the image. In general, a good encryption scheme can make the encrypted image has a uniform histogram to resist any statistical attacks. The original image and its histogram are shown in Fig.13 (a) and Fig. 13(b). Fig. 13(c) and Fig. 13(d) depict the encrypted image and corresponding histogram, respectively. As we can see, the histogram of the encrypted image is very uniform and is significantly different from that of the original image, which means that the encryption scheme based on the proposed MWCS can resist statistical attacks well.

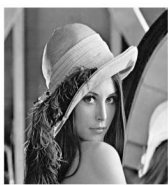

(a)

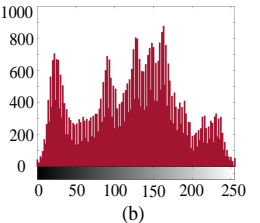

(b)

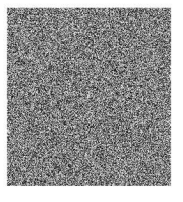

(c)

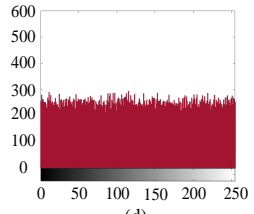

Fig. 13: Simulation results of the proposed encryption scheme. (a) Original image. (b) Histogram of the original image. (c) Encrypted image. (d) Histogram of the encrypted image.

2) Correlation analysis: The adjacent pixels of a original image have a strong correlation in the horizontal, vertical, and diagonal directions. But in a encrypted image, the correlation coefficients are expected to be close to zero in three directions. The correlation of each pair of pixels can be calculated by [35-36]

$$
\rho_{x y}=\frac{\sum_{i=1}^{N}\left(x_{i}-E(x)\right)\left(y_{i}-E(y)\right)}{\sqrt{\sum_{i=1}^{N}\left(x_{i}-E(x)\right)^{2}} \sqrt{\sum_{i=1}^{N}\left(y_{i}-E(y)\right)^{2}}},
$$

where $x$ and $y$ denote the intensity values of two adjacent pixels, $N$ is the total number of pixels, and $\mathrm{E}(\mathrm{x})$ and $\mathrm{E}(\mathrm{y})$ are the mean values of $x$ and $y$, respectively. As an example, we selected five pairs of adjacent pixels from the original image in horizontal directions, namely, $N=5,\left(x_{1}-x_{5}\right)=(93$, $105,110,106,103)$ and $\left(y_{1}-y_{5}\right)=(99,106,109,106,107)$. In this case, the correlation coefficient of the adjacent pixels in the original image can be calculated by Equation (12), namely $\rho_{x y}=0.960175$. After the original image is encrypted, we obtained the corresponding pixels from the encrypted image, namely $\left(x_{1}-x_{5}\right)=(126,93,109,56,65)$ and $\left(y_{1}-y_{5}\right)=(76,196$, $147,108,20$ ), respectively. Under this case, the correlation coefficient of the adjacent pixels in the encrypted image can be calculated by Equation (12), $\rho_{x y}=0.260651$. It shows that the correlation coefficients of the adjacent pixels in the original image are large, but there are low correlation coefficients after the original image is encrypted. It should be pointed that to obtain a more effective correlation coefficient, a larger $N$ should be selected. So, we randomly select 10000 pairs of adjacent pixels from the original image and the encrypted image in the horizontal, vertical, and diagonal directions to test their internal correlations. The correlation coefficients of the original and encrypted images are given in Table. III. It shows that the correlation coefficients of the original image are very close to 1 , while those of the encrypted image is around 0 in all directions. Therefore, the presented encrypted algorithm can effectively resist statistical attacks.

3) Entropy analysis: The information entropy is an important indicator in evaluating the randomness of a encrypted image. If an encryption scheme can generate encrypted images owning the maximum information entropy close to 8 , it means that it has excellent randomness property. In experiments, we can obtain the information entropy by calculating the following formula [5]

$$
H(m)=-\sum_{i=1}^{L} P\left(m_{i}\right) \log _{2}^{P\left(m_{i}\right)},
$$

where $m$ represents the type of all existing pixels in the image, the number of types is $L . P\left(m_{i}\right)$ denotes the probability value of the $m_{i}$ type pixel in the image. In grayscale images, $m_{i} \in(0$, $1,2,3, \ldots, 255), L=256, P\left(m_{i}\right) \in[0,1]$, and $\operatorname{sum}\left(P\left(m_{i}\right)\right)=1$. And the theoretical maximum information entropy is 8 . The closer the information entropy is to 8 , the more random the grayscale distribution of image pixels. For example, we assumes that there are only five types of the pixel in a gray image $256 \times 256$, namely $m_{1}-m_{5}$. Their probability values are $0.1,0.15,0.2,0.25$, and 0.3 , respectively. The information entropy of the image can be calculated by Equation (13), namely $\mathrm{H}=2.2282$. It means that the pixel distribution in this image is not uniform, that is, it has poor randomness property. Here, the information entropies of the original image and encrypted image are calculated using Equation (13), and the calculated entropy values of the original image and encrypted image are 0.0084 and 7.9976, respectively. It is found that there is a large improvement in information entropy after the original image is encrypted. Moreover, the entropy value of the encrypted image is close to the ideal value 8 , which shows that the proposed algorithm has a strong ability for improving information entropy.

4) Sensitivity analysis: The key sensitivity is an important indicator in evaluating the precision of a cryptosystem. In our encryption algorithm, $a, b, c, x_{0}, y_{0}$, and $z_{0}$ are all used as secret keys. In our decryption experiment, we choose $c, x_{0}$, and $z_{0}$ as the secret keys. When $c=0.45, x_{0}=0.3$, and $z_{0}=-$ 14, Fig. 14(a) illustrates the accurate decrypted image. Fig. 
14(b)-(d) illustrate the inaccurate decrypted image with a tiny change of $c, x_{0}$, and $z_{0}$, respectively. As can be seen, even the secret key is changed a little $\left(10^{-9}\right)$, the decrypted image is absolutely different from the original image. Therefore, the key sensitivity test shows that the proposed encryption scheme has perfect sensitivity to the key.

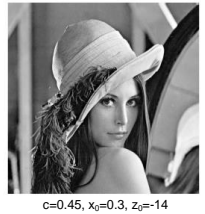

(a)

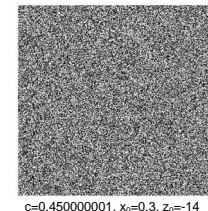

(b)

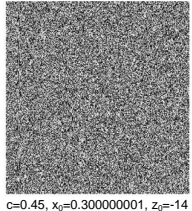

(c)

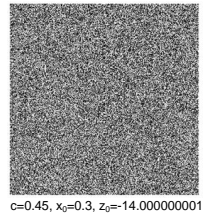

(d)
Fig. 14: Image decryption process with the secret keys $c, x_{0}$, and $z_{0}$. (a) Accurate decrypted image with the secret keys $c=0.45, x_{0}=0.3$, and $z_{0}=-14$. (b) Inaccurate decrypted image with the secret keys $c=0.450000001, x_{0}=0.3$, and $z_{0}=-14$. (c) Inaccurate decrypted image with the secret keys $c=0.45$, $x_{0}=0.300000001$, and $z_{0}=-14$. (d) Inaccurate decrypted image with the secret keys $c=0.45, x_{0}=0.3$, and $z_{0}=-14.000000001$.

Meanwhile, Table III presents the performance comparison of encryption results between encryption schemes based on different multi-wing chaotic systems (MWCSs) or multi-scroll chaotic systems (MSCSs). In Table III, the second column represents the type of original image, and the third column denotes the chaotic system used in the encryption schemes. The fourth column clearly demonstrates that the information entropy of the image generated from the proposed encryption scheme is higher than the information entropy of the image generated from other encryption schemes in Refs [13-14, $28,35]$. It shows that the proposed encryption scheme has better performance in terms of information entropy. And the sixth column in Table III demonstrates that the horizontal correlation (HC) coefficient of the encrypted image in this work is less than those in Refs. [13, 28, 35] and close to that in Ref. [14], and the vertical correlation (VC) coefficient of the encrypted image in this work is less than those in Refs. [13, 35], and close to that in Ref. [14, 28], and the diagonal correlation (DC) coefficient of the encrypted image in this work is less than those in Refs. [13-14, 35, 28]. These results demonstrate that our encryption scheme has a better ability to disrupt the correlation between adjacent pixels of an image. According to the above analysis, the presented encryption scheme based on MWCS can more effectively resist the entropy attack and statistical attacks and can be applied to protect image data in information communication.

\section{FPGA-BASEd CHAOTIC IMAGE CRYPTOSySTEM}

Hardware implementation is fundamental for chaos application in various engineering fileds. Although analog circuits can produce chaotic attractors, it is very difficult to establish an experimental chaotic communication hardware system on the analog electronic circuit platform due to the parasitic parameters of circuit components. In recent years, the application of FPGA in the field of chaotic secure communication provides strong technical support [38-39]. However, FPGA implementation of a chaotic system requires a lot of hardware resources, and only some simple chaotic systems can use this method. To the best of our knowledge, as traditional MWCSs have complex system models and circuit structures, there is no FPGA-based image encryption scheme based on MWCSs until now.

\section{A. Design of the FPGA-based Chaotic Image Cryptosystem}

In this section, we design and implement a MWCS-based image cryptosystem on the FPGA platform. The hardware architecture of the image cryptosystem is designed in Fig. 15, which mainly contains five parts: image RMA (random access memory), chaotic sequence controller, image encryption module, image decryption module, and VGA display controller. Among them, the image RMA is used to store and output the original image, and the chaotic sequence controller completes the generation and caching of chaotic sequences. For the process of image encryption, the original image and chaotic sequences are sent to the image encryption module synchronously. Then the original image is encrypted in the image encryption module, the encrypted image is sent to the VGA display controller and finally displays on the monitor. For the process of image decryption, the encrypted image and chaotic sequences are sent to the image decryption module synchronously. After that, the encrypted image is decrypted in the image decryption module, and the decrypted image is displayed on the monitor by the VGA display controller.

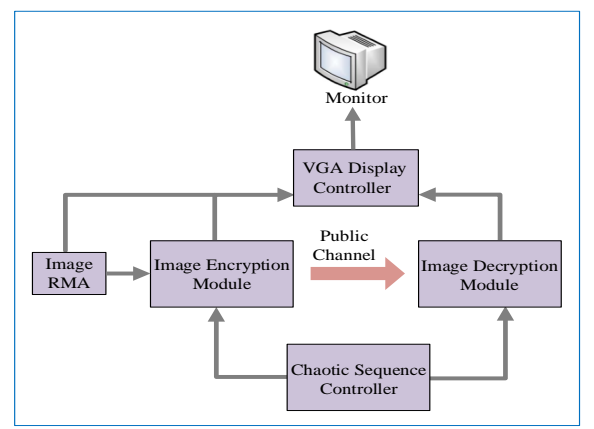

Fig. 15: Block diagram of FPGA-based image encryption and decryption.

\section{B. FPGA-based Implementation Results for Chaotic Image Cryptosystem}

Based on the designed hardware architecture in Fig. 15, the MWCS-based image cryptosystem is implemented by using a Xilinx Virtex-6 FPGA development board with a 32 bit IEEE 754-1985 floating-point standard. The functions of the five parts in Fig. 15 are realized by Verilog HDL programming, where the generation process of the chaotic sequences in a chaotic sequence controller can refer to our previous work in [38]. Before the experiment, because the output of the VGA display controller is a 24-bit RGB value, we first use the software 'Image2 $\mathrm{Lcd}^{\prime}$ to convert the original $256 \times 256$ 'Lena' $^{\prime}$ image from an 8-bit depth grayscale image to a 24-bit depth image. In the experiment, images are stored in the RAM of the ZYNQ-XC7Z020 chip. The FPGA development board and the experimental results are shown in Fig. 16. In Fig.16(b), the original image and the encrypted image are displayed on the monitor. When the image decryption module gets the correct key, the encrypted image can be correctly decrypted, as shown in Fig.16(c). On the contrary, when the decryption module gets an incorrect key, the encrypted image cannot be decrypted, as shown in Fig. 16(d). From the encryption and decryption results of three groups of images implemented by the FPGA hardware cryptosystem, there is no intuitive difference between the encryption effect and the MATLAB encryption simulation result, and the encrypted image can achieve the effect of simulation experiment in MATLAB. And the decrypted image can restore the original image well, 
TABLE III: PERFORMANCE COMPARISON OF ENCRYPTION SCHEMES BASED ON DIFFERENT CHAOTIC SYSTEMS

\begin{tabular}{|c|c|c|c|c|c|c|}
\hline Refs & $\begin{array}{l}\text { Image } \\
\text { type }\end{array}$ & $\begin{array}{l}\text { System } \\
\text { type }\end{array}$ & Entropy & $\begin{array}{c}\text { Correlation coefficients } \\
\text { HC, VC, DC (Original image) }\end{array}$ & $\begin{array}{c}\text { Correlation coefficients } \\
\text { HC, VC, DC (Encrypted image) }\end{array}$ & $\begin{array}{c}\text { Hardware } \\
\text { demonstration }\end{array}$ \\
\hline 2019[13] & Lena $(256 \times 256)$ & MSCS & 7.9889 & $0.9233,0.9672,0.8949$ & $-0.0062,0.0061,-0.0277$ & No \\
\hline $2020[35]$ & Lena $(256 \times 256)$ & MSCS & 7.9975 & $0.949,0.907,0.8795$ & $-0.0327,-0.0414,-0.0037$ & No \\
\hline $2019[28]$ & Lena $(256 \times 256)$ & MWCS & 1 & I, $0.8952,0.9583$ & I, $0.003821,0.006359$ & No \\
\hline $2020[14]$ & Lena $(256 \times 256)$ & MWCS & 7.9974 & $0.94505,0.96653,0.91917$ & $0.000682,-0.00077,-0.003636$ & No \\
\hline This work & Lena $(256 \times 256)$ & MWCS & 7.9976 & $0.972807,0.939337,0.921438$ & $0.000827,0.005238,-0.000455$ & Yes \\
\hline
\end{tabular}

which contains almost all the information and details of the original image. Furthermore, the time for encryption and decryption on the FPGA hardware cryptosystem is $0.262144 \mathrm{~s}$ and $0.262058 \mathrm{~s}$, respectively. This time is largely lower than the corresponding time 0.876952 s and 0.630132 s in MATLAB simulation. It shows that the chaotic cryptosystem implemented by FPGA can greatly improve the speed of data processing and the time of algorithm operation. Therefore, these experimental results validate the feasibility and reliability of the proposed MWCS-based encryption scheme. It should be pointed out that the maximum image size that can be encrypted is determined by the size of the block RAM. In this FPGA, the capacity of the block RAM is $5040 \mathrm{~kb}$.

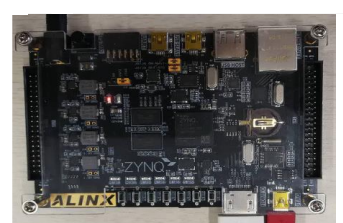

(a)

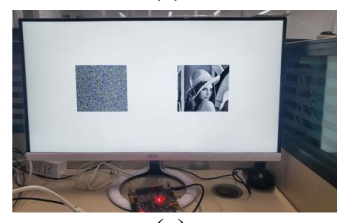

(c)

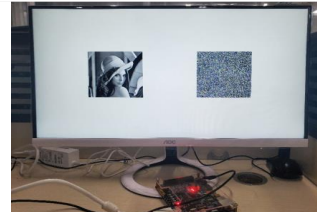

(b)

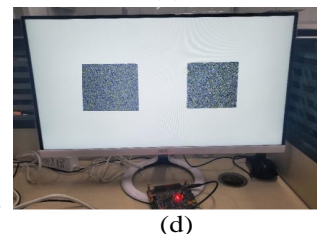

(d)
Fig. 16: FPGA-based implementation results of MWCSbased image cryptosystem. (a) Virtex-6 FPGA development board. (b) The original and encrypted image on the hardware platform. (c) The encrypted and correctly decrypted image on the hardware platform. (d) The encrypted and incorrectly decrypted image on the hardware platform.

\section{CONCLUSION}

The complex system model makes chaotic systems difficult to apply in various engineering fields. The main goal of the current study was to break the restriction of polynomial functions in MWCSs. This paper proposed a simple MWCS without polynomial functions. We have affirmed that the MWCS can generate multi-butterfly attractors with an arbitrary number of butterflies. The number of butterflies can be regulated by changing the self-oscillation time, control parameters, or initial states. The MWCS model has been verified by an analog circuit. Performance evaluations clarify that, compared with the existing MWCSs, the circuit implementation of the MWCS is greatly simplified due to its simple system model. Moreover, the proposed MWCS was applied to design a chaotic image encryption scheme. Simulation results show that the MWCS-based image encryption scheme has better security properties than those image encryption scheme based on existing MWCSs. And the image encryption scheme has been implemented on the FPGA platform. Experimental results further validated the effectiveness and feasibility of the MWCS. Therefore, the MWCS is suitable for chaosbased engineering applications. Certainly, a weakness of this MWCS is that with the increase of wings the more time will be expended. Adjusting the frequency and amplitude of the sinusoidal function may be a good method to solve this problem, which is what we need to study in the future.

\section{REFERENCES}

[1] R. M. May, "Simple mathematical models with very complicated dynamics," Nature., vol. 261, no. 5560, pp. 459-467, 1976.

[2] S. H. Luo and Y. D. Song, "Chaos analysis-based adaptive backstepping control of the microelectromechanical resonators with constrained output and uncertain time delay," IEEE Trans. Ind. Electron., vol. 63, no. 10, pp. 6217-6225, 2016.

[3] Hua, Z., Zhou, B., and Zhou, Y, "Sine chaotification model for enhancing chaos and its hardware implementation," IEEE Trans. Ind. Electron., vol. 66, no. 2, pp. 1273-1284, 2019.

[4] Q. Wang, S. Yu, C. Li, J. Lu, X. Fang, C. Guyeux, and J. M. Bahi, "Theoretical design and FPGA-based implementation of higherdimensional digital chaotic systems," IEEE Trans. Circuits Syst. I-Regul. Pap., vol. 63, no. 3, pp. 401-412, 2016.

[5] S. Wang, C. Wang, and C. Xu, "An image encryption algorithm based on a hidden attractor chaos system and the Knuth-Durstenfeld algorithm," Opt. Lasers Eng., vol. 128, Art. no. 105995, 2020.

[6] E. N. Lorenz, "Deterministic nonperiodic flow," J. Atmos. Sci., vol. 20, no. 2 , pp. $130-141,1963$

[7] Hua, Z., Zhou, B., and Zhou, Y, "Sine-transform-based chaotic system with FPGA implementation," IEEE Trans. Ind. Electron., vol. 65, no. 3, pp. 2557-2566, 2018

[8] H. Lin, C. Wang, W. Yao, and Y. Tan, "Chaotic dynamics in a neural network with different types of external stimuli," Commun. Nonlinear Sci. Numer. Simul., vol. 90, Art. no. 105390, 2020.

[9] Q. Hong, Q. Wu, X. Wang, and Z. Zeng, "Novel nonlinear function shift method for generating multiscroll attractors using memristor-based control circuit," IEEE Trans. Very Large Scale Integr. (VLSI) Syst., vol. 27, no. 5, pp. 1174-1185, 2019.

[10] C. Volos, J. Maaita, S. Vaidyanathan, V. Pham, I. Stouboulos, and I. Kyprianidis, "A novel four-dimensional hyperchaotic four-wing system with a saddle-focus equilibrium," IEEE Trans. Cir. Sys.-II: Brief Papers., vol. 64, no. 3, pp. 339-343, 2017.

[11] L. S. Ramya, R. Sakthivel, C. K. Ahn, and Y. Ren, "Reliable resilienttime control for stabilization of hyperchaotic fractional-order systems," IEEE Trans. Cir. Sys.-II: Brief Papers., vol. 66, no. 9, pp. 1537-1541, 2019.

[12] H. Lin, C. Wang, Q. Hong, Y. Sun, "A multi-stable memristor and its application in a neural network," IEEE Trans. Cir. Sys.-II: Brief Papers., 2020, doi: 10.1109/TCSII.2020.3000492.

[13] N. Wang, C. Li, H. Bao, M. Chen, and B. Bao, "Generating multiScroll chua's attractors via simplified piecewise-linear Chua's diode," IEEE Trans. Circuits Syst. I-Regul. Pap., vol. 66, no. 12, pp. 4767-4779, 2019.

[14] F. Yu, L. Liu, S. Qian, et al, "Chaos-based application of a novel multistable 5D memristive hyperchaotic system with coexisting multiple attractors," Complexity., vol. 2020, Art. no. 8034196, 2020.

[15] A. S. Elwakil, S. Ozoguz, and M. P. Kennedy, "Creation of a complex butterfly attractor using a novel Lorenz-type system," IEEE Trans. Circuits Syst. I-Regul. Pap., vol. 49, no. 4, pp. 527-530, 2002.

[16] W. Liu and G. Chen, "A new chaotic system and its generation," Int. J. Bifurcation Chaos., vol. 13, no. 1, pp. 261-267, 2003.

[17] F. Yu, L. Liu, H. Shen, et al, "Multistability analysis, coexisting multiple attractors and FPGA implementation of Yu-Wang four-wing chaotic system," Math. Probl. Eng., vol. 2020, Art. no. 7530976, 2020.

[18] J. Ma, L. Wang, S. Duan, and Y. Xu, "A multi-wing butterfly chaotic system and its implementation," Int. J. Circuit Theory Appl., vol. 45, no. 11, pp. 1873-1884, 2017.

[19] S. Yu, W. K. S. Tang, J. Lu, and G. Chen, "Generation of $n \times m$-wing Lorenz-like attractors from a modified shimizu-morioka model," IEEE Trans. Cir. Sys.-II: Brief Papers., vol. 55, no. 1, pp. 1168-1172, 2008.

[20] S. Yu, J. L $\ddot{u}$, G. Chen, and X. Yu, "Design and implementation of grid multiwing butterfly chaotic attractors from a piecewise Lorenz system," IEEE Trans. Cir. Sys.-II: Brief Papers., vol. 57, no. 10, pp. 803-807, 2010.

[21] S. Yu, J. Lü, X. Yu, and G. Chen, "Design and implementation of grid multiwing hyperchaotic Lorenz system family via switching control and constructing super-heteroclinic loops," IEEE Trans. Circuits Syst. I-Regul. Pap., vol. 59, no. 5, pp. 1015-1028, 2012.

[22] F. R. Tahir, S. Jafari, V. Pham, C. K. Volos, and X. Wang, "A novel no-equilibrium chaotic system with multiwing butterfly attractors," Int. J. Bifurcation Chaos., vol. 25, no. 4, Art. no. 1550056, 2015. 
[23] Y. Huang, P. Zhang, and W. Zhao, "Novel grid multiwing butterfly chaotic attractors and their circuit design," IEEE Trans. Cir. Sys.-II: Brief Papers., vol. 62, no. 5, pp. 496-500, 2015.

[24] Q. Lai, X. Zhao, K. Rajagopal, G. Xu, A. Akgul, and E. Guleryuz, "Dynamic analyses, FPGA implementation and engineering applications of multi-butterfly chaotic attractors generated from generalised Sprott C system," Pramana., vol. 90, no. 1, Art. no. 6, 2018.

[25] L. Zhou, C. Wang, and L. Zhou, "Generating hyperchaotic multi-wing attractor in a 4D memristive circuit," Nonlinear Dyn., vol. 85, no. 4, pp. 2653-2663, 2016.

[26] Q. Xie, Y. Zeng, "Generating different types of multi-double-scroll and multi-double-wing hidden attractors," Eur. Phys. J.-Spec. Top., vol. 229, pp. 1361-1371, 2020

[27] N. Yu, Y. Wang, X. Liu, and J. Xiao, "3D grid multi-wing chaotic attractors," Int. J. Bifurcation Chaos., vol. 28, no. 4, Art. no. 1850045 2018

[28] Q. Hong, Y. Li, X. Wang, and Z. Zeng, "A versatile pulse control method to generate arbitrary multidirection multibutterfly chaotic attractors," IEEE Trans. Comput-Aided Des. Integr. Circuits Syst., vol. 38, no. 8 , pp. 1480-1492, 2019.

[29] Q. Wu, Q. Hong, X. Liu, X. Wang, and Z. Zeng, "A novel amplitude control method for constructing nested hidden multi-butterfly and multiscroll chaotic attractors," Chaos Solitons Fractals., vol. 134, Art. no. 109727, 2020.

[30] J.C. Sprott, "Some simple chaotic flows," Phys. Rev. E., vol. 50, no. 2 , pp. 647-650, 1994

[31] M. Chen, M. Sun, H. Bao, Y. Hu, and B. Bao, "Flux-charge analysis of two-memristor-based Chua' s circuit: dimensionality decreasing mode for detecting extreme multistability," IEEE Trans. Ind. Electron., vol. 67, no. 3, pp. 2197-2206, 2020.

[32] H. Bao, Z. Hua, N. Wang, et al, "Initials-boosted coexisting chaos in a 2D sine map and its hardware implementation," IEEE Trans. Ind. Informat., 2020. doi: 10.1109/TII.2020.2992438.

[33] C. Wang, H. Xia, and L. Zhou, "A memristive hyperchaotic multiscrol Jerk system with controllable scroll numbers," Int. J. Bifurcation Chaos. vol. 27, Art. no. $1750091,2017$.

[34] Z. Hua, Y. Zhou, and B. Bao, "Two-dimensional sine chaotification system with hardware implementation," IEEE Trans. Ind. Informat., vol. 16, no. 2, pp. 887-897, 2020.

[35] T. Wang, L. Song, M. Wang, and Z. Zhuang, "A novel image encryption algorithm based on parameter-control scroll chaotic attractors," IEEE Access., vol. 8, pp. 36281-36292, 2020.

[36] F. Özkaynak, "Brief review on application of nonlinear dynamics in image encryption,"Nonlinear Dyn., vol. 92, no. 2, pp. 305-313, 2018

[37] C. Xu, J. Sun, and C. Wang "An image encryption algorithm based on random walk and hyperchaotic systems," Int. J. Bifurcation Chaos., vol. 30, Art. no. 2050060, 2020.

[38] F. Yu, L. Liu, Z, B. He, et al, "Analysis and FPGA realization of a novel 5D hyperchaotic four-wing memristive system, active control synchronization, and secure communication application," Complexity. vol. 2019, Art. no. 4047957, 2019.

[39] R. F. Molanes, J. J. Rodriguez-Andina, and J. Farina, "Performance characterization and design guidelines for efficient processor-FPGA communication in cyclone V FPSoCs," IEEE Trans. Ind. Electron., vol. 65, no. 5, pp. 4368-4377, 2018.

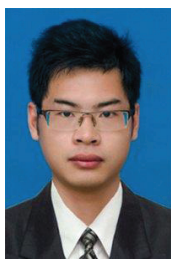

Hairong Lin received the M.S. degree in information and communication engineering from $\mathrm{Hu}-$ nan University, Changsha, China, in 2015. He is currently working toward the Ph.D degree at the College of Computer Science and Electronic Engineering, Hunan University, Changsha, China. His main research interest includes nonlinear systems and circuits, memristive neuromorphic circuit, and artificial intelligence.

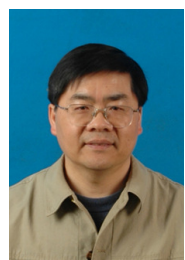

Chunhua Wang received the M.S. degree from Zhengzhou University, Zhengzhou, China, in 1994 and the Ph.D. degree from Beijing University of Technology, Beijing, China, in 2003. He is currently the Professor of College of Information Science and Engineering, Hunan University, Changsha, China. $\mathrm{He}$ is the Doctor tutor, director of advanced communication technology key laboratory of hunan universities, the member of academic committee of hunan university, the director of chaos and nonlinear circuit professional committee of circuit and system branch of China electronic society. Now, his research interests include memristor circuit, complex networks, chaotic circuit, chaos secure communication, current-mode circuit and neural networks based on memristor. $\mathrm{He}$ has presided over 8 national and provincial projects, and published more than 120 papers, among which more than 100 were retrieved by SCI.

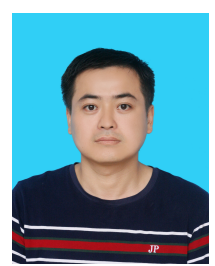

Fei Yu the M.E. and Ph.D. degree from College of Information Science and Engineering, Hunan University, Changsha, China, in 2010 and 2013, respectively. He is currently a distinguished associate professor at School of Computer and Communication Engineering in Changsha University of Science and Technology, Changsha, China. He focuses on nonlinear system and circuit, complex network and their applications.

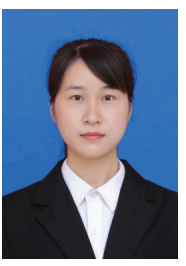

Cong $\mathrm{Xu}$ received the Bachelor's degree in communications engineering from Hunan City University, Yiyang, China. She is currently pursuing the $\mathrm{Ph}$. D degree in College of Computer Science and Electronic Engineering, Hunan University, China. He research interests include memristor neural network and its applications.

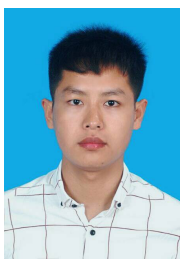

Qinghui Hong received the Ph.D degree in computer system architecture from Huazhong University of Science and Technology, Wuhan, China, in 2019. $\mathrm{He}$ is currently an Assistant Professor with college of computer science and electronic engineering, Hunan University, Changsha 410082, China. His current research interests include memristive neural network and its application to Artifificial Intelligence.

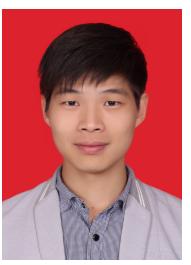

Wei Yao received the M.S. degree from College of Information Science and Engineering, Hunan University, Changsha, China, in 2014. He is currently pursuing the $\mathrm{Ph} . \mathrm{D}$ degree in Hunan University. His research interests mainly focus on nonlinear dynamical systems, complex systems and complex networks.

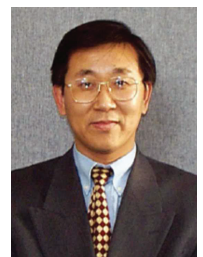

Yichuang Sun (M'90-SM'99) received the B.Sc. and M.Sc. degrees from Dalian Maritime University, Dalian, China, in 1982 and 1985, respectively, and the Ph.D. degree from the University of York, York, U.K, in 1996, all in communications and electronics engineering.

Dr. Sun is currently Professor of Communications and Electronics, Head of Communications and Intelligent Systems Research Group, and Head of Electronic, Communication and Electrical Engineering Division in the School of Engineering and Computer Science of the University of Hertfordshire, UK. He has published over 330 papers and contributed 10 chapters in edited books. He has also published four text and research books: Continuous-Time Active Filter Design (CRC Press, USA, 1999), Design of High Frequency Integrated Analogue Filters (IEE Press, UK, 2002), Wireless Communication Circuits and Systems (IET Press, 2004), and Test and Diagnosis of Analogue, Mixed-signal and RF Integrated Circuits - the Systems on Chip Approach (IET Press, 2008). His research interests are in the areas of wireless and mobile communications, $\mathrm{RF}$ and analogue circuits, microelectronic devices and systems, and machine learning and deep learning.

Professor Sun was a Series Editor of IEE Circuits, Devices and Systems Book Series (2003-2008). He has been Associate Editor of IEEE Transactions on Circuits and Systems I: Regular Papers (2010-2011, 2016-2017, 2018 2019). He is also Editor of ETRI Journal, Journal of Semiconductors, and Journal of Sensor and Actuator Networks. He was Guest Editor of eight IEEE and IEE/IET journal special issues: High-frequency Integrated Analogue Filters in IEE Proc. Circuits, Devices and Systems (2000), RF Circuits and Systems for Wireless Communications in IEE Proc. Circuits, Devices and Systems (2002), Analogue and Mixed-Signal Test for Systems on Chip in IEE Proc. Circuits, Devices and Systems (2004), MIMO Wireless and Mobile Communications in IEE Proc. Communications (2006), Advanced Signal Processing for Wireless and Mobile Communications in IET Signal Processing (2009), Cooperative Wireless and Mobile Communications in IET Communications (2013), Software-Defined Radio Transceivers and Circuits for $5 \mathrm{G}$ Wireless Communications in IEEE Transactions on Circuits and Systems-II (2016), and the 2016 IEEE International Symposium on Circuits and Systems in IEEE Transactions on Circuits and Systems-I (2016). He has also been widely involved in various IEEE technical committee and international conference activities. 